\title{
The central melanocortin system affects the hypothalamo- pituitary thyroid axis and may mediate the effect of leptin
}

\author{
M.S. Kim, C.J. Small, S.A Stanley, D.G.A. Morgan, L.J. Seal, W.M. Kong, \\ C.M.B. Edwards, S. Abusnana, D. Sunter, M.A. Ghatei, and S.R. Bloom \\ Endocrine Unit, Imperial College School of Medicine, Hammersmith Hospital, London W12 0NN, United Kingdom \\ Address correspondence to: S.R. Bloom, Endocrine Unit, Imperial College School of Medicine, Hammersmith Hospital, \\ Du Cane Road, London W12 0NN, United Kingdom. \\ Phone: 44-181-383-3242; Fax: 44-181-383-3142; E-mail: s.bloom@ic.ac.uk.
}

Received for publication November 4, 1999, and accepted in revised form February 22, 2000.

\begin{abstract}
Prolonged fasting is associated with a downregulation of the hypothalamo-pituitary thyroid (H-P-T) axis, which is reversed by administration of leptin. The hypothalamic melanocortin system regulates energy balance and mediates a number of central effects of leptin. In this study, we show that hypothalamic melanocortins can stimulate the thyroid axis and that their antagonist, agouti-related peptide (Agrp), can inhibit it. Intracerebroventricular (ICV) administration of Agrp (83-132) decreased plasma thyroid stimulating hormone (TSH) in fed male rats. Intraparaventricular nuclear administration of Agrp (83-132) produced a long-lasting suppression of plasma TSH, and plasma T4. ICV administration of a stable $\alpha-\mathrm{MSH}$ analogue increased plasma TSH in 24-hour-fasted rats. In vitro, $\alpha-\mathrm{MSH}$ increased thyrotropin releasing hormone (TRH) release from hypothalamic explants. Agrp (83-132) alone caused no change in TRH release but antagonized the effect of $\alpha$-MSH on TRH release. Leptin increased TRH release from hypothalami harvested from 48-hour-fasted rats. Agrp (83-132) blocked this effect. These data suggest a role for the hypothalamic melanocortin system in the fasting-induced suppression of the H-P-T axis.
\end{abstract}

J. Clin. Invest. 105:1005-1011 (2000).

\section{Introduction}

Energy intake and expenditure are tightly regulated in mammals (1). It has been shown that starvation is associated with a depression of the thyroid axis. Thus, triiodothyronine (T3), thyroxine (T4), thyroid stimulating hormone (TSH), and hypothalamic thyrotropin releasing hormone (TRH) are reduced in humans and rodents during starvation $(2,3)$. The resultant decrease in energy expenditure is likely to be an important adaptive response to diminished energy supplies. Leptin signals nutritional status to the hypothalamus. In particular, plasma leptin concentrations decrease with fasting (4). Administration of leptin during fasting prevents the suppression of the hypothalamo-pituitary thyroid (H-P-T) axis $(5,6)$.

Agouti-related peptide (Agrp) is an endogenous antagonist at the melanocortin 3 and 4 receptor (MC3-R and MC4-R) (7), at which the proopiomelanocortin (POMC) products, such as $\alpha$-melanocyte stimulating hormone $(\alpha$ $\mathrm{MSH}$ ), act as agonists. POMC and Agrp cell bodies are located within the hypothalamic arcuate nucleus (7). The hypothalamic melanocortin system is an essential regulator of food intake and energy homeostasis (8). Mutations of POMC and MC4-R in human and mouse are associated with gross obesity (9-11). Hypothalamic Agrp mRNA and the density of MC4-R have been shown to change with altered nutritional states in the rodent $(12,13)$.

Recently, triple-labeling fluorescent immunohistochemistry with $\alpha$-MSH, Agrp, and TRH antisera has been used to demonstrate that both $\alpha-\mathrm{MSH}$ and Agrp axonal terminals are closely apposed to TRH-producing cell bodies and dendrites in the hypothalamic paraventricular nucleus (PVN) (14). This suggests that PVN TRH neurons could be under dual regulation by Agrp and $\alpha$-MSH. Thus, we hypothesize that the hypothalamic melanocortin system may have a role in regulation of the H-P-T axis. The hypothalamic melanocortin system is an established mediator of leptin's effects on feeding (15), brown adipose tissue uncoupling protein1 (via the sympathetic nervous system) $(16,17)$, and gonadal function (18). We, therefore, also hypothesize that the melanocortin system may be a potential mediator of leptin's actions on the H-P-T axis.

To test this hypothesis, we investigated whether (a) hypothalamic Agrp and $\alpha$-MSH altered the H-P-T axis in the rat; (b) the effects of both peptides on the H-P-T axis changed with nutritional status; and (c) antagonism of the melanocortin system by Agrp blocked the effect of leptin on the H-P-T axis.

\section{Methods}

Peptides. Agrp (83-132) was purchased from Phoenix Pharmaceuticals Inc. (Mountain View, California, USA). $\alpha$-MSH and [Nle $\left.{ }^{4}, \mathrm{D}-\mathrm{Ph} \mathrm{e}^{7}\right]-\alpha \mathrm{MSH}$ (NDP-MSH) were purchased from Peninsula (St. Helens, Merseyside, United Kingdom). Leptin was obtained from R\&D Systems Inc. (Abingdon, Oxford, United Kingdom).

Animals. Male Wistar rats (specific pathogen free; Imperial College School of Medicine, London, United 

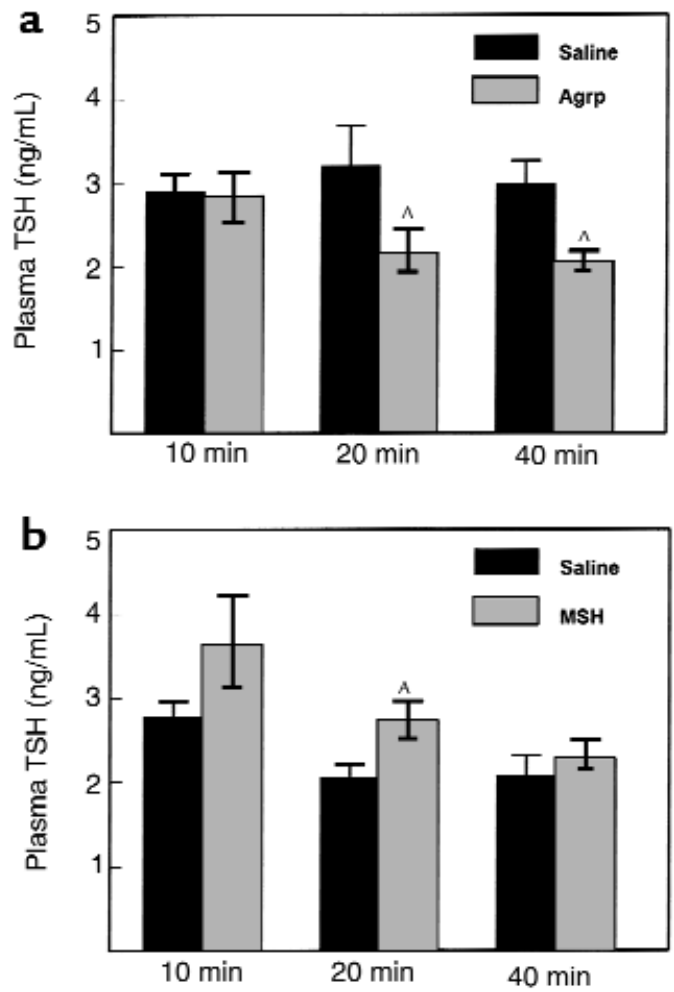

Figure 1

The effects of (a) ICV Agrp (83-132) (2 nmol) on plasma TSH in fed rats and (b) ICV NDP-MSH (5 nmol) on plasma TSH in 24hour-fasted rats. Either peptide or saline was administered to the rat $3 r d$ ventricle in the early light phase (0900-1100 hours). Plasma TSH concentration was measured at 10, 20, and 40 minutes after injection. ${ }^{A} P<0.05$ versus saline-injected control group.

Kingdom) weighing 250-300 g were maintained in individual cages under controlled temperature $\left(21-23^{\circ} \mathrm{C}\right)$ and light (12-hour light/dark cycle; lights on at 0700 hours), with ad libitum access to food (RM1 diet; SDS Ltd., Witham, United Kingdom), unless described in experimental protocols, and water.

RIA. Plasma TSH levels were assayed using methods and reagents provided by A. Parlow (NIDDK National Hormone and Pituitary Program, Baltimore, Maryland, USA). Plasma total T4 was measured using a solid-phase RIA (Diagnostic Products Corp., Los Angeles, California, USA). The TRH concentration in aCSF was assayed using methods and reagents provided by $\mathrm{H}$. Fraser (Medical Research Council, Edinburgh, United Kingdom) (19). The $\alpha$-MSH RIA was developed using an antibody from Chemicon International Inc. (Temecula, California, USA). The cross-reactivity with $\beta, \gamma_{1}, \gamma_{2}, \gamma_{3}-\mathrm{MSH}$, and ACTH was less than $0.01 \%$, and the detection limit of the assay was $0.5 \mathrm{fmol} /$ tube.

ICV or intraparaventricular nuclear cannulation and injection. Animals were implanted with permanent 22-gauge (ICV) or 26-gauge (iPVN) stainless steel cannulae (Plastics One Inc., Roanoke, Virginia, USA) into the third cerebral ventricle (ICV) or into the paraventricular nucleus (iPVN), as described previously $(20,21)$. After a 7 day-recovery period, animals were handled daily for 1 week to minimize the stress response. All compounds were dissolved in $0.9 \%$ saline and administered in a volume of $10 \mu \mathrm{L}$ (ICV) or $1 \mu \mathrm{l}$ (iPVN). For ICV cannulated animals, the correct position of the cannula was confirmed by a positive dipsogenic response to angiotensin II $(150 \mathrm{ng})$. The correct cannula placement of iPVN cannulae was confirmed by histological examination after studies were completed. Only those animals with correctly positioned cannulae, independently assessed, were included in the analysis of data.

After injection, animals were sacrificed by decapitation, and trunk blood was collected in plastic lithium heparin tubes containing $0.6 \mathrm{mg}$ aprotinin. Plasma was separated by centrifugation and stored at $-20^{\circ} \mathrm{C}$. Plasma TSH concentration was measured by RIA.

Static incubation of hypothalamic explants. The hypothalamic explants (1.8-mm-thick slice taken parallel to the base of the hypothalamus) were maintained in a static incubation system using artificial cerebrospinal fluid (aCSF) gassed with $95 \% \mathrm{O}_{2}$ and $5 \% \mathrm{CO}_{2}$, as described
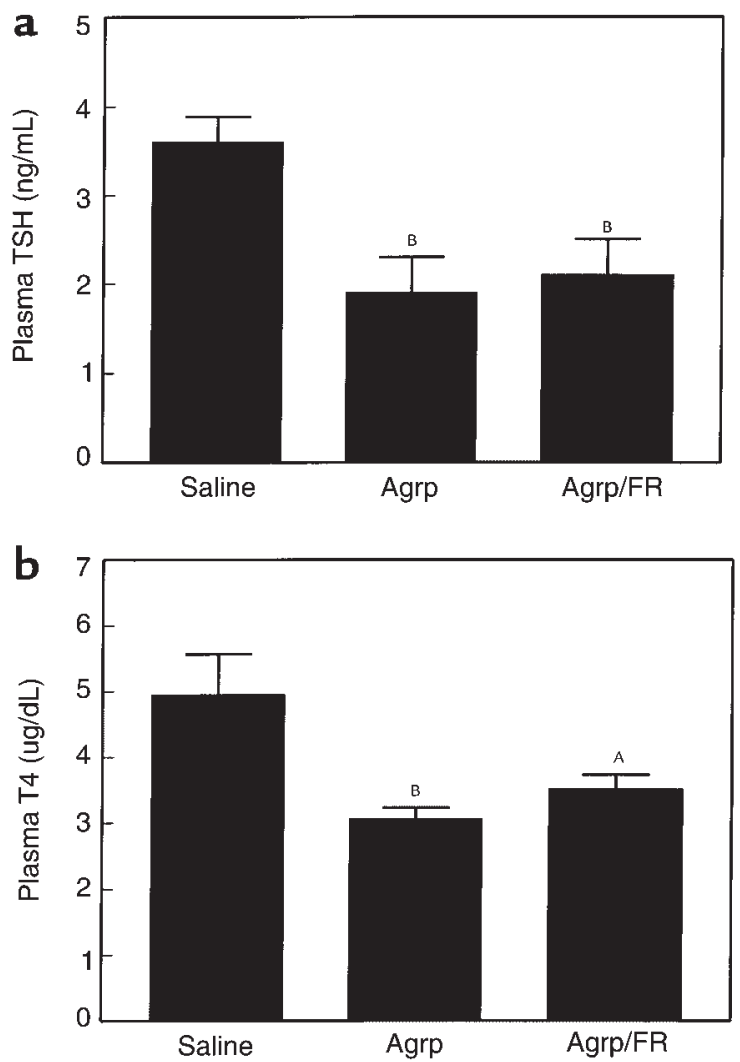

Figure 2

The effect of iPVN Agrp (83-132) on (a) plasma TSH and (b) plasma T4. Agrp ( $0.1 \mathrm{nmol})$ or saline was administered into the rat PVN in the beginning of the dark phase (1900-2000 hours). Plasma TSH and $\mathrm{T} 4$ concentrations were measured at the end of the dark phase, 15 hours after injection (0900-1100 hours). Three groups were investigated: saline; (saline injected and ad libitum fed), Agrp (Agrp injected and ad libitum fed), Agrp/FR (Agrp-injected and foodrestricted $[F R]$ to saline control group). ${ }^{A} P<0.01$ versus saline-injected group, ${ }^{B} P<0.005$ versus saline-injected group. 
previously (22). After an initial 2-hour equilibration period, the hypothalami were incubated for 45 minutes in $500 \mu \mathrm{L}$ aCSF (basal period) before being challenged with the test peptide in $500 \mu \mathrm{L}$ aCSF for 45 minutes (test period). Finally, the viability of the tissue was verified by 45 minutes of exposure to $56 \mathrm{mM} \mathrm{KCl}$ solution (potassium period). At the end of each period, aCSF was collected and stored at $-20^{\circ} \mathrm{C}$ before measurement of TRH or $\alpha$-MSH by RIA. The hypothalamic explants that showed less than $200 \%$ of basal release in response to 56 $\mathrm{mM} \mathrm{KCl}$ solution were excluded in the data analysis.

Statistical analysis. Results are presented as mean \pm SEM. Statistical differences between control and experimental group in the in vivo studies were determined by ANOVA, followed by post hoc Least Significance Difference (Systat, Evanston, Illinois, USA). Data from hypothalamic explant experiment were compared by paired $t$ test between basal and test period. In all cases, $P<0.05$ was considered to be statistically significant.

\section{Results}

Study 1(a). Effect of ICV administration of Agrp (83-132) on plasma TSH in fed or 24-hour-fasted animals. To investigate whether an antagonist of melanocortin receptors affected the H-P-T axis, a biologically active $\mathrm{COOH}$-terminal fragment of Agrp (2 nmol Agrp [83-132]) or saline was administered ICV to fed or 24-hour-fasted animals ( $n=10-12$ per group) during the early light phase (0800-1100 hours). This dose of Agrp (83-132) was previously found to produce a significant increase in food intake (23). Plasma TSH was measured at 10, 20, and 40 minutes after injection.

Fed animals. Agrp (83-132) significantly decreased plasma TSH at 20 minutes after injection (Agrp $2.1 \pm$ $0.3 \mathrm{ng} / \mathrm{mL}$ vs. saline $3.1 \pm 0.5 \mathrm{ng} / \mathrm{mL} ; P<0.05$; Figure 1a), and this effect was sustained at 40 minutes (Agrp $2.0 \pm 0.3 \mathrm{ng} / \mathrm{mL}$ vs. saline $3.0 \pm 0.1 \mathrm{ng} / \mathrm{mL} ; P<0.05)$.

Fasted animals. Agrp (83-132) did not cause a significant change in plasma TSH at any time point (20 minutes; Agrp $2.3 \pm 0.3 \mathrm{ng} / \mathrm{mL}$ vs. saline $2.2 \pm 0.1 \mathrm{ng} / \mathrm{mL} ; P=\mathrm{NS}$ ).

Study 1(b). Effect of ICV administration of NDP-MSH on plasma TSH in fed or 24-hour-fasted animals. To investigate the effect of melanocortin receptor agonism on the $\mathrm{H}$ P-T axis, NDP-MSH (a stable analogue of $\alpha-\mathrm{MSH}, 5$ $\mathrm{nmol}$ ) or saline was administered ICV to fed or 24hour-fasted animals ( $n=10-13$ per group) during the early light phase. This dose of NDP-MSH was found to produce a $50 \%$ reduction in food intake (our unpublished observations). Plasma TSH was measured at 10, 20, and 40 minutes after injection.

Fed animals. NDP-MSH did not significantly alter plasma TSH levels at any time point (20 minutes; NDP-MSH $5 \mathrm{nmol} 3.0 \pm 0.2 \mathrm{ng} / \mathrm{mL}$ vs. saline $3.6 \pm 0.4 \mathrm{ng} / \mathrm{mL} ; P=\mathrm{NS}$ ).

Fasted animals. NDP-MSH significantly increased plasma TSH at 20 minutes (NDP-MSH $2.7 \pm 0.2 \mathrm{ng} / \mathrm{mL}$ vs. saline $2.0 \pm 0.2 \mathrm{ng} / \mathrm{mL} ; P<0.05$; Figure $1 \mathrm{~b})$.

Study 2. Effect of iPVN administration of Agrp (83-132) on plasma TSH and total T4 Agrp induces a long-lasting increase in food intake when administered centrally
$(23,24)$. To investigate whether the effect of Agrp on the H-P-T axis is dependent on food intake, 3 groups of PVN cannulated animals were investigated $(n=11-13$ per group). In group 1 , animals received saline and were ad libitum fed. In group 2, animals were Agrp injected and ad libitum fed. In group 3, animals received Agrp and were food restricted to ensure the same weight of food was eaten as the saline control group. Agrp (83132) ( $0.1 \mathrm{nmol})$ or saline was administered directly into the PVN at the beginning of the dark phase (lights off at 1900 hours). Previously, this dose of Agrp (83-132) increased 24-hour food intake by $200 \%$ in PVN cannulated animals (24). At the end of the dark phase, 15 hours after injection, animals were decapitated and trunk blood was collected. Plasma TSH and total T4 were measured by RIA.

IPVN Agrp (83-132) significantly decreased plasma TSH in both the ad libitum and food-restricted groups (saline, $3.4 \pm 0.3 \mathrm{ng} / \mathrm{mL} ;$ Agrp/ad libitum, $1.9 \pm 0.4 \mathrm{ng} / \mathrm{mL}$; Agrp/food-restricted, $2.1 \pm 0.4 \mathrm{ng} / \mathrm{mL}$; both groups, $P<$ 0.005 vs. saline; Figure 2a) and plasma total T4 (saline, 4.9 $\pm 0.6 \mu \mathrm{g} / \mathrm{dL} ;$ Agrp/ad libitum, $3.1 \pm 0.2 \mu \mathrm{g} / \mathrm{dL} ; P<0.005 \mathrm{vs}$. saline; Agrp/food-restricted, $3.5 \pm 0.2 \mu \mathrm{g} / \mathrm{dL} ; P<0.01$ vs. saline; Figure 2b) 15 hours after injection.

Study 3. Effect of $\alpha$-MSH or/and Agrp (83-132) on TRH release from bypothalami harvested from fed and 48hour-fasted animals. To investigate the effect of $\alpha-\mathrm{MSH}$
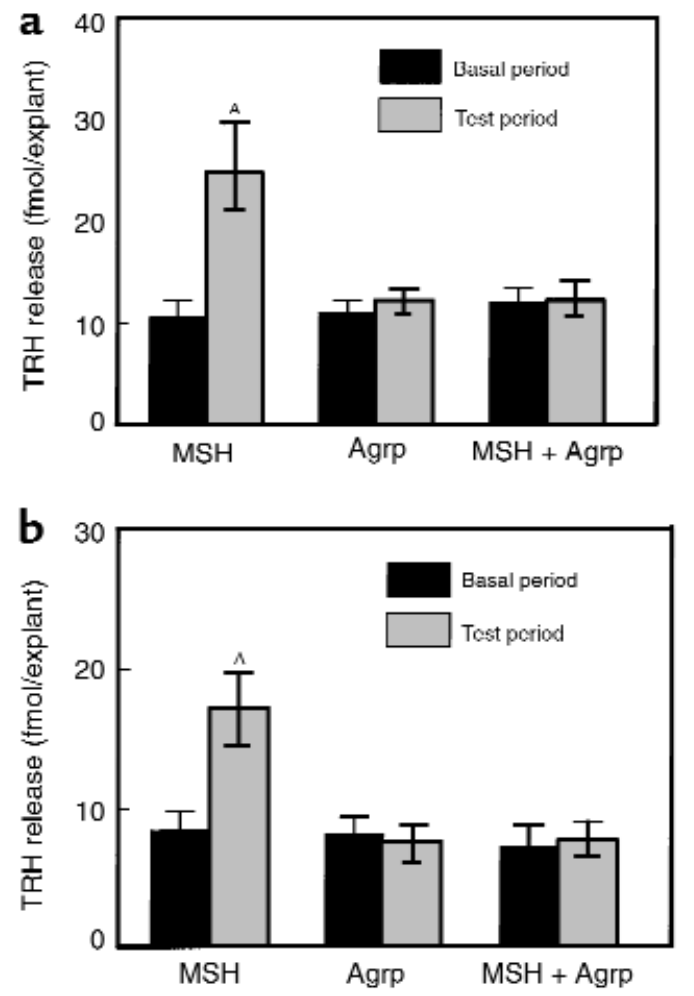

Figure 3

The effects of $\alpha-\mathrm{MSH}$ (100 nM), Agrp (83-132) (100 nM), and both $\alpha-\mathrm{MSH}$ (100 nM) and Agrp (83-132) (100 nM) on TRH release from hypothalamic explants harvested from (a) fed animals and (b) 48hour-fasted animals. ${ }^{A} P<0.01$ versus basal period. 
a

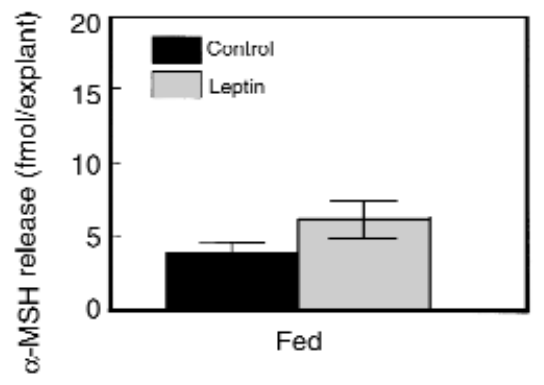

c

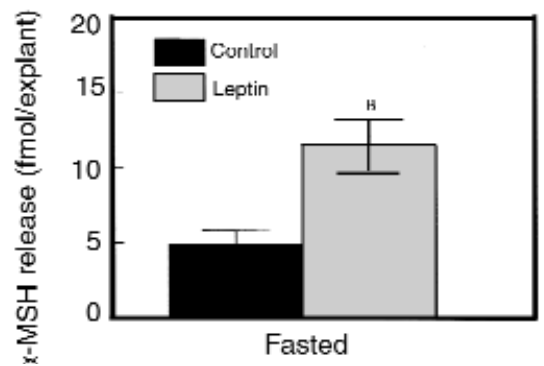

b

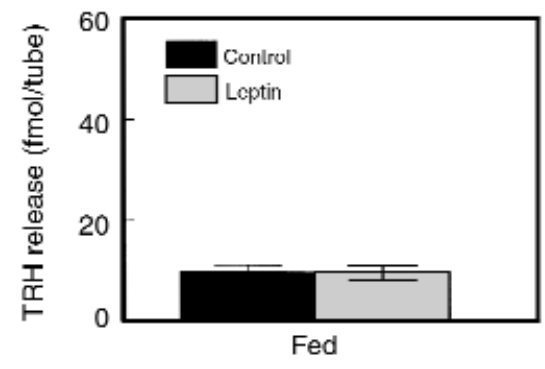

d

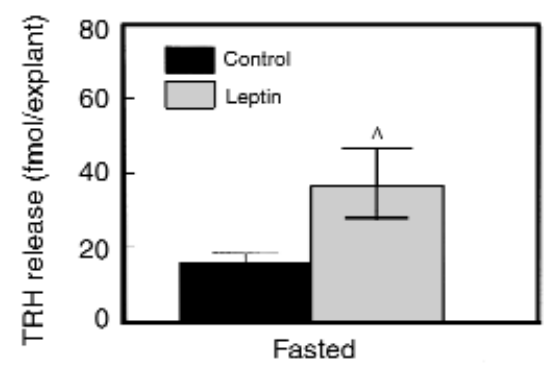

Figure 4

The effect of leptin (100 $\mathrm{nM}$ ) on $\alpha-\mathrm{MSH}$ release $(\mathbf{a}, \mathbf{c})$ and TRH release $(\mathbf{b}, \mathbf{d})$ from the hypothalami harvested from fed rats (a, $\mathbf{b})$ and 48 hour-fasted rats $(\mathbf{c}, \mathbf{d})$. ${ }^{A} P<0.05$ versus basal period. ${ }^{\mathrm{B}} P<0.005$ versus saline.

and Agrp on TRH release in vitro, hypothalamic explants harvested from fed and 48-hour-fasted animals were incubated with $\alpha$-MSH (100 nM), Agrp (83132) $(100 \mathrm{nM})$, or both $\alpha-\mathrm{MSH}$ and Agrp (83-132) $(n=$ 15 per group).

Fed animals. $\alpha$-MSH significantly increased TRH release from hypothalamic explants (basal, $10.6 \pm 1.7$ $\mathrm{fmol} /$ explant; $\alpha$-MSH-treated, $24.8 \pm 4.8 \mathrm{fmol} /$ explant; $P<0.01$; Figure 3a). Agrp (83-132) alone did not alter TRH release (basal, $11.1 \pm 1.2 \mathrm{fmol} /$ explant; Agrp-treated ,12.2 $\pm 1.2 \mathrm{fmol} /$ explant; $P=\mathrm{NS}$; Figure $3 \mathrm{a}$ ) but blocked the effect of $\alpha-\mathrm{MSH}$ (basal, $12.1 \pm 1.4$ fmol/explant; Agrp $+\alpha$-MSH, $12.3 \pm 1.8$ fmol/explant; $P=$ NS; Figure 3a).

Fasted animals. Comparable effects were observed in hypothalamic slices harvested from 48-hour-fasted animals. Again, $\alpha-\mathrm{MSH}$ significantly increased TRH release (basal, $8.4 \pm 1.4 \mathrm{fmol} /$ explant; $\alpha$-MSH-treated, $17.2 \pm 2.5 \mathrm{fmol} /$ explant; $P<0.01$; Figure $3 \mathrm{~b})$. Agrp (83132) alone did not alter TRH release (basal, $8.2 \pm 1.2$ fmol/explant; Agrp-treated, $7.6 \pm 1.2 \mathrm{fmol} /$ explant; $P=$ $\mathrm{NS}$; Figure $3 \mathrm{~b}$ ) but blocked the effect of $\alpha-\mathrm{MSH}$ (basal, $7.3 \pm 1.4 \mathrm{fmol} /$ explant; Agrp $+\alpha-\mathrm{MSH}, 7.8 \pm 1.2$ fmol/explant; $P=\mathrm{NS}$; Figure $3 \mathrm{~b}$ ).

Study 4. Effect of leptin on $\alpha M S H$ and TRH release from bypothalami harvested from fed or 48-hour-fasted animals. To compare the effect of leptin on $\alpha$-MSH and TRH release in fed and 48-hour-fasted animals, hypothalamic explants were incubated with leptin $(100 \mathrm{nM})(n=$ 15 per group).

Fed animals. Leptin did not significantly increase secretion of either $\alpha$-MSH (basal, $4.0 \pm 0.7$ fmol/explant; leptin-treated, $6.3 \pm 1.2 \mathrm{fmol} /$ explant; $P$ = 0.06; Figure 4a) or TRH (basal, $9.8 \pm 1.4 \mathrm{fmol} /$ explant; leptin-treated $9.9 \pm 1.4 \mathrm{fmol} /$ explant; $P=\mathrm{NS}$; Figure $4 \mathrm{~b}$ ) from hypothalamic explants.

Fasted animals. Leptin significantly increased secretion of both $\alpha$-MSH (basal, $4.9 \pm 0.9 \mathrm{fmol} /$ explant; leptintreated, $11.5 \pm 1.7 \mathrm{fmol} /$ explant; $P<0.005$; Figure $4 \mathrm{c}$ ) and TRH (basal, $15.8 \pm 2.6 \mathrm{fmol} /$ explant; leptin-treated, $36.5 \pm 10.3 \mathrm{fmol} /$ explant; $P<0.05$; Figure $4 \mathrm{~d}$ ) from hypothalamic explants.

Study 5. Blocking effect of Agrp (83-132) on leptin-induced TRH release from bypothalami harvested from 48-hour-fasted animals. The results of study 4 demonstrated that leptin had a significant stimulatory effect on $\alpha-\mathrm{MSH}$ and TRH release from hypothalamic explants harvested from 48-hour-fasted animals. To investigate the role of the melanocortin system in mediating the leptin effect on TRH release, we examined the ability of Agrp (83-132) to block leptin-induced TRH release. Leptin (100 nM), Agrp (83-132) (100 nM) or both leptin and Agrp (83-132) were applied to hypothalamic explants harvested from 48-hour-fasted animals $(n=$ 10 per group).

Leptin significantly increased TRH release from the hypothalami harvested from 48-hour-fasted rats (basal, $13.5 \pm 2.2 \mathrm{fmol} /$ explant; leptin-treated, $40.6 \pm 10.2$ fmol/explant; $P<0.05$; Figure 5). Agrp (83-132) alone did not alter TRH release (basal, $10.9 \pm 1.0 \mathrm{fmol} / \mathrm{explant}$; Agrp (83-132)-treated; $12.3 \pm 2.3 \mathrm{fmol} /$ explant; $P=\mathrm{NS}$; Figure 5) but blocked the leptin-stimulated TRH release (basal, $12.1 \pm 1.8 \mathrm{fmol} /$ explant; leptin + Agrp. $18.4 \pm 4.5$ fmol/explant; $P=$ NS; Figure 5). 


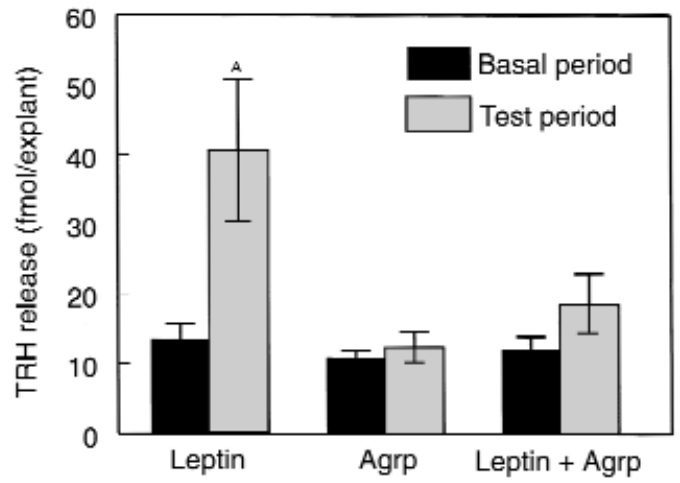

Figure 5

The effect of leptin (100 nM), Agrp (83-132) (100 nM), and both leptin (100 nM) and Agrp (83-132) (100 nM) on TRH release from hypothalami harvested from 48 -hour-fasted rats. ${ }^{A} P<0.05$ versus basal period.

\section{Discussion}

In this study, central administration of Agrp (83-132) to rats significantly decreased plasma TSH and total T4, whereas NDP-MSH increased plasma TSH. Consistent with these findings, $\alpha$-MSH increased TRH release from hypothalamic explants and Agrp (83-132) blocked the $\alpha-\mathrm{MSH}$-induced increase in TRH release.

The hypothalamic melanocortin system has been shown to respond to changes in nutritional status (12, 13). For example, Agrp mRNA increases by 10 - to 15 fold after a 48-hour-fast (12), and POMC mRNA has been reported to decrease in response to fasting (25). We have shown here that the effects of exogenous Agrp (83132) and NDP-MSH on the H-P-T axis are also dependent on feeding status. ICV Agrp (83-132) decreases plasma TSH in the fed state but not in the fasted state, whereas, conversely, NDP-MSH increases plasma TSH in the fasted state but not in the fed state. However, in isolated hypothalamic explants from both fed and fasted animals, TRH release is increased in response to $\alpha$ MSH, but Agrp (83-132) alone has no effect.

The following model, illustrated in Figure 6, may explain these findings. In the fasted state, circulating leptin is low (4). This decreases hypothalamic POMC (25) and, thus, the melanocortin receptor agonist $\alpha$ $\mathrm{MSH}$, and increases Agrp (12). Thus, melanocortin receptor activation is likely to be at a low level in the fasted state. Hence, exogenous Agrp had no significant effect, as there was little endogenous melanocortin receptor activation for it to block. When exogenous NDP-MSH was administered to fasted animals, it was able to increase melanocortin receptor activation and to increase plasma TSH.

In the fed state, circulating leptin is high, POMC is high, and Agrp is low. Hence, melanocortin receptor activation would be at a high level. Under these circumstances, exogenous NDP-MSH was without effect, as melanocortin receptors were already activated, but exogenous Agrp was able to block this receptor activation, and reduced plasma TSH.
In our in vitro experiments, $\alpha$-MSH increased TRH release from hypothalamic explants from both fed and fasted animals, suggesting that melanocortin receptors are not fully activated. Agrp alone had no effect on the slices, further suggesting a low level of melanocortin receptor activation. It is thus possible that the isolation of the hypothalamus from extra hypothalamic inputs causes a loss of melanocortin tone. This phenomenon of loss of melanocortin tone in hypothalamic explants has also been observed by another group. They demonstrated that SHU9119 blocked synaptic responses stimulated by exogenous MT II in hypothalamic slices

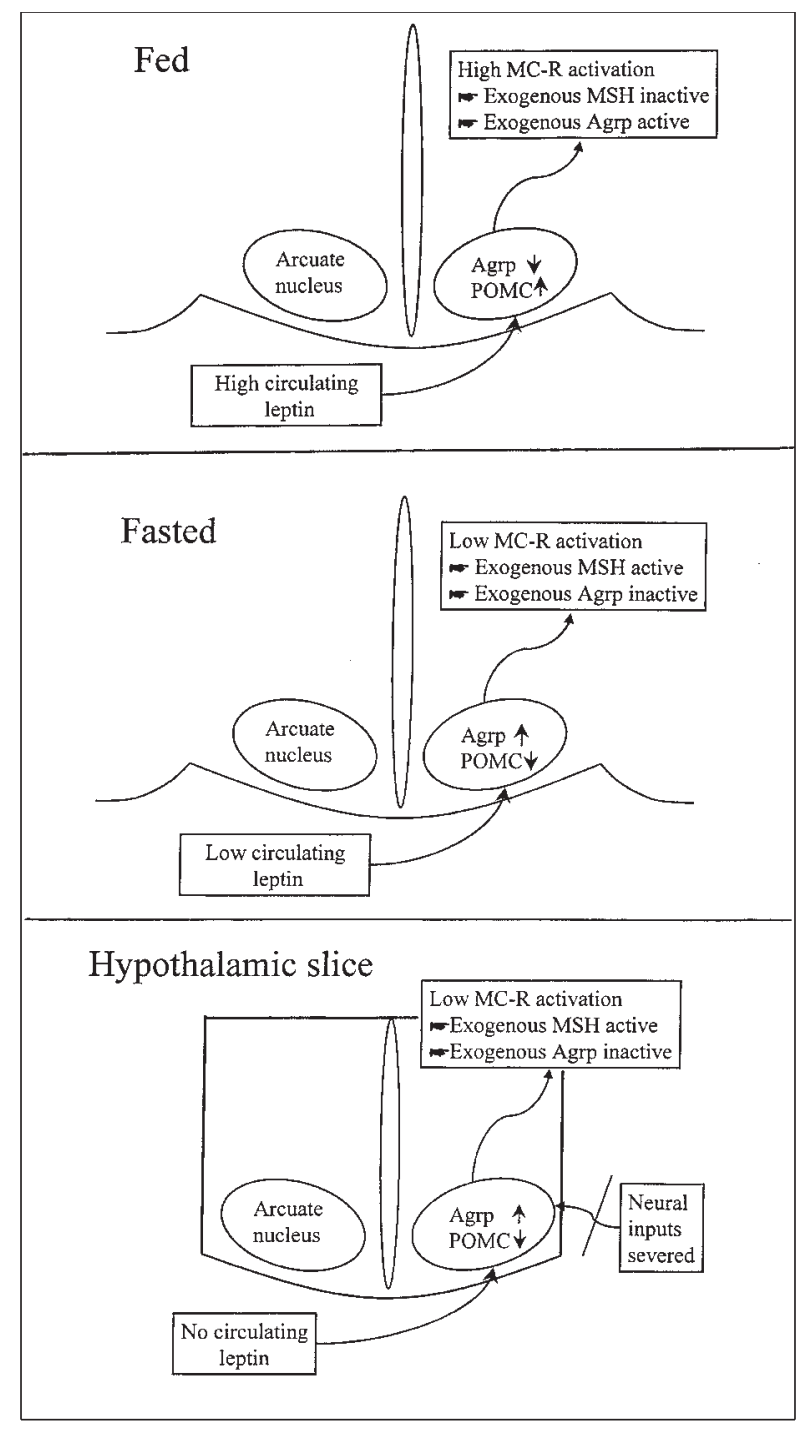

Figure 6

A hypothetical model showing changes of hypothalamic POMC, Agrp, and melanocortin receptor activation in fed animals, fasted animals, and hypothalamic slices. In the fed state, high level of circulating leptin increases POMC and decreases Agrp, resulting in high activation of melanocortin receptor. In this situation, exogenous $\alpha$ $\mathrm{MSH}$ is inactive and Agrp is active. In the fasted state and in the hypothalamic slice, low or no circulating leptin induces changes of the central melanocortin system, low POMC, high Agrp, and low melanocortin receptor activation. In this situation, exogenous $\alpha$ $\mathrm{MSH}$ is active and Agrp is inactive. 
including the PVN, but had no effect alone, whereas in vivo, SHU9119 increased food intake when injected into the rat PVN (26).

This model, based on our results, suggests that hypothalamic melanocortin tone is maintained by feedingdependent, extrahypothalamic inputs. One possible input is circulating leptin.

Fasting induces a suppression of PVN proTRH mRNA expression and depresses the H-P-T axis $(2,3)$. This is reversed by administration of leptin $(5,6)$. A number of central effects of leptin are mediated by melanocortins (15-18). Hence, we investigated whether the hypothalamic melanocortin system could mediate the effects of leptin on the H-P-T axis. Leptin caused a significant increase in TRH release from hypothalamic explants harvested from 48 -hour-fasted rats. Leptin also caused an increase in $\alpha$-MSH release from these slices. Although Agrp (83-132) had no effect on basal TRH release, it was able to reverse the stimulatory effect of leptin on TRH release. This suggests that increased $\alpha-\mathrm{MSH}$ release mediates at least part of leptin's actions on the H-P-T axis.

Although leptin increased $\alpha-\mathrm{MSH}$ and TRH release from hypothalamic explants from 48-hour-fasted rats, no significant effect was seen on explants from fed animals. This difference may be explained by the well-documented increase in arcuate nucleus leptin receptors in fasting $(27,28)$. Hence, hypothalamic explants from fasted animals would be more sensitive to exogenous leptin.

ProTRH mRNA expression in the PVN changes with metabolic state (6). Pro-TRH neurons here are densely innervated by both $\alpha-\mathrm{MSH}$ and Agrp neuronal projections from the arcuate nucleus $(14,29)$. TRH neurons in the lateral hypothalamus are neither responsive to metabolic status nor innervated with melanocortin neurons $(6,29)$. To test whether PVN TRH neurons were responsive to melanocortins, Agrp (83-132) was directly injected into the PVN animals. This produced a long-lasting suppression of plasma TSH with a simultaneous fall in plasma $\mathrm{T} 4$, which was independent of Agrp-stimulated food intake. Thus, it would appear that PVN TRH neurons are tonically stimulated by melanocortin agonists in the fed state.

A role for the arcuate nucleus mediating the effects of fasting and leptin on the H-P-T axis is suggested by previous data. After ablation of the arcuate nucleus, and therefore of POMC- and Agrp-containing neurons, there was no fasting-induced suppression of the H-P-T axis and no effect of exogenous leptin (30). As well as the melanocortin-mediated effect of leptin, there could be a direct effect of leptin on the TRH neuron. Recently, it has been reported that leptin stimulates TRH promoter activity in primary cultures of rat fetal hypothalamic neurons (31).

Although the data presented in this article suggest a role for the melanocortins in the fasting-induced suppression of the H-P-T axis, this does not preclude the involvement of other hypothalamic peptides. Neuropeptide Y (NPY) has been described as a possible hypothalamic mediator of leptin actions on the H-P-T axis (32). NPY and Agrp are produced by the same arcuate neurons (33). Immunohistochemical investigation demonstrates that nerve terminals immunoreactive for NPY and Agrp, as well as for $\alpha$ $\mathrm{MSH}$, are found in close proximity to PVN TRH neurons $(18,30,33)$. The NPY knockout mouse has a normal H-P$T$ response to fasting (34). It will be interesting to examine whether prolonged fasting induces a suppression of the H-P-T axis in the POMC- and MC4-R-deficient mice.

Our data has suggested a role of the melanocortin/Agrp system in the central regulation of thyroid function and provided evidence that it may act as a mediator of leptin's actions on the H-P-T axis. This system may provide a link between the control of food intake and energy expenditure.

\section{Acknowledgments}

We thank the Medical Research Council (MRC) for program grant support (G7811974). S.A. Stanley, L.J. Seal, and W.M. Kong are Wellcome Trust clinical training fellows. D. Sunter is funded by an MRC PhD studentship.

1. Silva, J.E. 1995. Thyroid hormone control of thermogenesis and energy balance. Thyroid. 5:481-492.

2. Blake, N.G., Eckland, D.J., Foster, O.J., and Lightman, S.L. 1991. Inhibition of hypothalamic thyrotropin-releasing hormone messenger ribonucleic acid during food deprivation. Endocrinology. 129:2714-2718.

3. Spencer, C.A., Lum, S.M., Wilber, J.F., Kaptein, E.M., and Nicoloff, J.T. 1983. Dynamics of serum thyrotropin and thyroid hormone changes in fasting. J. Clin. Endocrinol. Metab. 56:883-888.

4. Weigle, D.S., et al. 1997. Effect of fasting, refeeding, and dietary fat restriction on plasma leptin levels. J. Clin. Endocrinol. Metab. 82:561-565.

5. Ahima, R.S., et al. 1996. Role of leptin in the neuroendocrine response to fasting. Nature. 382:250-252.

6. Legradi, G., Emerson, C.H., Ahima, R.S., Flier, J.S., and Lechan, R.M. 1997. Leptin prevents fasting-induced suppression of prothyrotropinreleasing hormone messenger ribonucleic acid in neurons of the hypothalamic paraventricular nucleus. Endocrinology. 138:2569-2576.

7. Ollmann, M.M., et al. 1997. Antagonism of central melanocortin receptors in vitro and in vivo by agouti-related protein. Science. 278:135-138.

8. Fan, W., Boston, B.A., Kesterson, R.A., Hruby, V.J., and Cone, R.D. 1997. Role of melanocortinergic neurons in feeding and the agouti obesity syndrome. Nature. 385:165-168

9. Krude, H., et al. 1998. Severe early-onset obesity, adrenal insufficiency and red hair pigmentation caused by POMC mutations in humans. Nat. Genet. 19:155-157.

10. Huszar, D., et al. 1997. Targeted disruption of the melanocortin-4 receptor results in obesity in mice. Cell. 88:131-141.

11. Vaisse, C., Clement, K., Guy, G.B., and Froguel, P. 1998. A frameshift mutation in human MC4R is associated with a dominant form of obesity. Nat. Genet. 20:113-114.

12. Mizuno, T.M., and Mobbs, C.V. 1999. Hypothalamic agouti-related protein messenger ribonucleic acid is inhibited by leptin and stimulated by fasting. Endocrinology. 140:814-817.

13. Harrold, J.A., Widdowson, P.S., and Williams, G. 1999. Altered energy balance causes selective changes in melanocortin-4 (MC4-R), but not melanocortin-3 (MC3-R), receptors in specific hypothalamic regions: further evidence that activation of MC4-R is a physiological inhibitor of feeding. Diabetes. 48:267-271.

14. Fekete, C., et al. 2000. $\alpha$-melanocyte-stimulating hormone is contained in nerve terminals innervating thyrotropin releasing hormone synthesizing neurons in the hypothalamic paraventricular nucleus and prevents fasting induced suppression of prothyrotropin releasing hormone gene expression. J. Neurosci. 20:1550-1558.

15. Seeley, R.J., et al. 1997. Melanocortin receptors in leptin effects. Nature. 390:349.

16. Satoh, N., et al. 1998. Satiety effect and sympathetic activation of leptin are mediated by hypothalamic melanocortin system. Neurosci. Lett. 249:107-110.

17. Haynes, W.G., Morgan, D.A., Djalali, A., Sivitz, W.I., and Mark, A.L. 1999. Interactions between the melanocortin system and leptin in control of sympathetic nerve traffic. Hypertension. 33:542-547.

18. Watanobe, H., Schioth, H.B., Wikberg, J.E.S., and Suda, T. 1999. The 
melanocortin 4 receptor mediates leptin stimulation of luteinizing hormone and prolactin surges in steroid-primed ovariectomized rats. Biochem. Biophys. Res. Commun. 257:860-864.

19. Akinsanya, K.O., Ghatei, M.A., and Bloom, S.R. 1995. Gonadal steroids regulate rat anterior pituitary levels of TSH releasing hormone and pyroglutamyl-glutamyl-proline amide like immunoreactivity. Endocrinology. 136:734-740.

20. O'Shea, D., et al. 1997. Neuropeptide Y induced feeding in the rat is mediated by a novel receptor. Endocrinology. 138:196-202.

21. Edwards, C.M., et al. 1999. The effect of the orexins on food intake: comparison with neuropeptide $\mathrm{Y}$, melanin-concentrating hormone and galanin. J. Endocrinol. 160:R7-R12.

22. Stanley, S.A., et al. 1999. Agouti related peptide (Agrp) stimulates the hypothalamo pituitary gonadal axis in vivo and in vitro in male rats. Endocrinology. 140:5459-5462.

23. Rossi, M., et al. 1998. A C-terminal fragment of Agouti-related protein increases feeding and antagonizes the effect of alpha-melanocyte stimulating hormone in vivo. Endocrinology. 139:4428-4431.

24. Kim, M.S., et al. 2000. Hypothalamic localization of the feeding effect of agouti-related peptide and $\alpha$-melanocyte stimulating hormone. Diabetes. 49:177-182.

25. Mizuno, T.M., et al. 1998. Hypothalamic pro-opiomelanocortin mRNA is reduced by fasting and in ob/ob and db/db mice, but is stimulated by leptin. Diabetes. 47:294-297.

26. Cowley, M.A., et al. 1999. Integration of NPY, AGRP, and melanocortin signals in the hypothalamic paraventricular nucleus: evidence of a cel- lular basis for the adipostat. Neuron. 24:155-163.

27. Baskin, D.G., et al. 1998. Increased expression of mRNA for the long form of the leptin receptor in the hypothalamus is associated with leptin hypersensitivity and fasting. Diabetes. 47:538-543.

28. Baskin, D.G., Breininger, J.F., Bonigut, S., and Millerm, M.A. 1999. Leptin binding in the arcuate nucleus is increased during fasting. Brain Res. 828:154-158.

29. Legradi, G., and Lechan, R.M. 1999. Agouti-related protein containing nerve terminals innervate thyrotropin-releasing hormone neurons in the hypothalamic paraventricular nucleus. Endocrinology. 140:3643-3652.

30. Legradi, G., et al. 1998. Arcuate nucleus ablation prevents fastinginduced suppression of ProTRH mRNA in the hypothalamic paraventricular nucleus. Neuroendocrinology. 68:89-97.

31. Nillni, E., et al. 1999. Leptin regulates proTRH biosynthesis. Proc. Am. Endocr. Soc. 81st Ann. Meet. OR36:1. (Abstr.)

32. Legradi, G., and Lechan, R.M. 1998. The arcuate nucleus is the major source for neuropeptide Y-innervation of thyrotropin-releasing hormone neurons in the hypothalamic paraventricular nucleus. Endocrinology. 139:3262-3270.

33. Broberger, C., Johansen, J., Johansson, C., Schalling, M., and Hokfelt, T. 1998. The neuropeptide Y/agouti gene-related protein (AGRP) brain circuitry in normal, anorectic, and monosodium glutamate-treated mice. Proc. Natl. Acad. Sci. USA. 95:15043-15048.

34. Erickson, J.C., Ahima, R.S., Hollopeter, G., Flier, J.S., and Palmiter, R.D. 1997. Endocrine function of neuropeptide Y knockout mice. Regul. Pept. 70:199-202. 